\title{
STUDY ON THE INTERDEPENDENCE OF THE HORIZONTAL- VERTICAL ILLUSION AND THE DIVIDED ILLUSION: I
}

\author{
YOJI SUTO
}

Tokyo Electrical Engineering College

The so-called horizontal-vertical illusion has been studied by many investigators. The different explanations and analyses have been made to throw light on the occurrence of the overestimation of the vertical line as compared with the horizontal line (Fröbes, 1917; Imai, 1960; Künapas, 1955; Ono, 1959; Woodworth, 1954). But it may be said that a sufficient explanation can not be found and the further experimental studies are required.

Recently Künnapas (1955) pointed out that the two different illusions appeared in the horizontal-vertical figures: (a) the classical overestimation of the vertical line as compared with a horizontal line of equal length, and (b) the overestimation of the dividing line. Künnapas made a considerable contribution to the consideration of the horizontal-vertical illusion. But the studies on the so-called horizontalvertical illusion have been performed by the following procedure. The observers are required to compare the lengths of the vertical line and the horizontal line, for example, in the figure $\perp$. It is undisputed that many studies by this procedure made the important contributions to the consideration of this illusion. But the following problem arises in the above figure. How the apparent length of the divided horizontal line varies? This problem has been generally neglected in the previous studies. So far as the experimental procedure mentioned above is employed, the variation of the apparent length of the divided horizontal line can not to be clarified. But in the majority of the figures used to study the horizontal- vertical illusion, the horizontal lines are divided by the vertical lines, and vice versa.

The present study starts from this problem and aims to measure the apparent lengths of both horizontal and vertical lines. By this procedure, the relation between the horizontal-vertical illusion and the illusion of the divided line will be analysed in the present study. It is the purpose of the present study to analyse and clarify the interdependence of both illusions in many figures, and to consider their occurrences. The study on the variation of the apparent length of the horizontal line, for example, in the figure $\perp$, may be called the study of the divided illusion which has also been investigated by many persons (Imai, 1960; Obonai, 1955). But an illusion of the length of a line divided by another line, an illusion which will be investigated in the present study, has been scarecely studied up to the present. For this reason, the present study is titled "Study on the Interdependence of the Horizontal-Vertical Illusion and the Divided Illusion". So the present study is characterized by analysing the appearances of both illusions under the identical condition.

\section{MethoD}

The figures, $1 \mathrm{~mm}$ wide, were drawn with

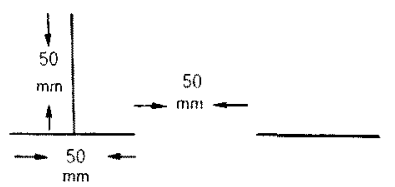

Fig. 1. black ink on the white cardboards. These are presented in the illuminated visual field. As 
Fig. 1 shows, a horizontal line is always presented on the right side of the visual field and this is used as a comparison line. The figures are presented on the left side, and a vertical line, a tilted line, or a horizontal line in the figures is used as a standard line.

The observers are required to observe binocularly and to adjust the length of the comparison line by the method of adjustment until both the standard and the comparison lines appear equal. Although the special instruction is not given, the observers are asked to observe with the naive attitude. The results are computed by the equation

$$
R E=\frac{E-E_{0}}{E_{0}} \times 100,
$$

where $E$ is the PSE obtained by observing the standard line, which is $50 \mathrm{~mm}$ in length and is a vertical, a tilted, or a horizontal line in the figures presented on the left side, and $E_{0}$ is the PSE obtained in the control experiment which is performed by presenting a horizontal line of $50 \mathrm{~mm}$ as the standard line on the left side. By this method, the amounts of apparent lengthening or shortening of different lines in many figures can be measured respectively.

The control experiment is performed at the intermediate position in the course of each experiment, for eliminating the anisotropy of the visual space. Two horizontal lines of the left and the right sides are presented at the same height, approximately at the height of the observer's eyes. The observer's head is held steady by he chin-rest. The illumination of the lighted surface on which stimulus figures are presented is 1400 lux. The distance between this surface and the observer's eyes is $110 \mathrm{~cm}$. Both ascending and descending series are repeated four times. The number of the observers varies from 4 to 6 depending upon the experimental series.

\section{EXPERIMENTS}

\section{Experiment 1}

Purpose. The purpose of this experiment is to clarify how the apparent lengths of the vertical line and the bisected horizontal line vary in the inverted $T$ figure with the variation of the position of the vertical

line.

Condition. The position of the vertical line in the figure shown in Fig. 2 is varied from the midpoint position of the horizontal line to its right end position.

Results and discussion. Average results for $4 \mathrm{Ss}$ are shown in Fig. 2. The abscissa of Fig. 2 indicates the position of the vertical line and $0 \mathrm{~cm}$ position means that the vertical is located at the midpoint of the horizontal. The ordinate indicates the relative effect of the overestimation or the underestimation of the lines. Plus means the relative effect of the overestimation and minus means the relative effect of the underestimation computed by the equation mentioned above.

As is shown in Fig. 2, the amount of overestimation of the vertical line de-

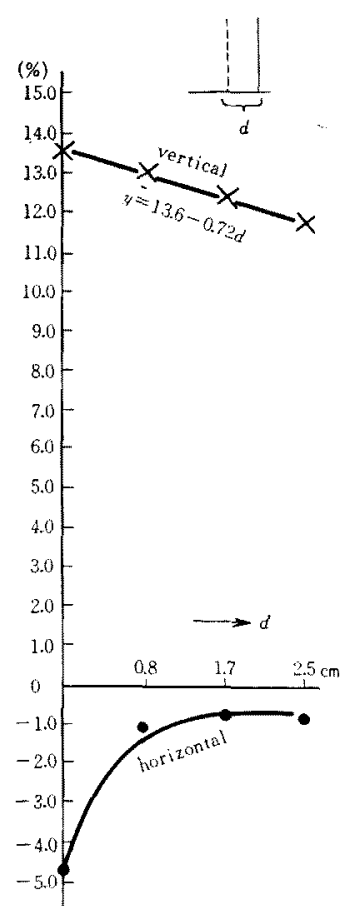

Fig. 2. The apparent lengths of the vertical and the horizontal line as functions of the position of the vertical line. divided line, $c$ is the constant, and $b$ and $c$ are experimentally determined. Assumcreases as the distance between the vertical line and the midpoint of the horizontal line increases. This result agrees with the confirmation of $\mathrm{Kün}$ napas (1955). The line in Fig. 2 is fitted by the method of least squares $\left(r_{y x}\right.$ $=-.994)$. Künnapas represented his result by the equation $x=\left(1-\frac{2 a}{d}\right) b+c$ where $x=$ the total illusion, $a=$ the distance of the dichosection from the midpoint, $\quad b=$ the maximal magnitude of the illusion in the midpoint position, $d$ =the whole length of the etermined. Assum- 
ing Künnapas' equation applicable to the present result, though his equation was obtained under the different situation from the present one, his equation was found considerably fit to the present result. But comparing his equation with the author's equation, indicated in Fig. 2, the sum of the squares of the residuals obtained by the author's regression equation is smaller than that obtained by $\mathrm{Kün}$ napas' equation (.021136:.10024 in relative amount of overestimation). So the present equation may be said more suitable than the Künappas' for the situation of the present study.

On the other hand, the horizontal line is underestimated and the amount of this underestimation decreases as the distance between the vertical line and the midpoint of the horizontal line increases. A significant difference was found between the results obtained under the conditions $d$ $=0 \mathrm{~cm}$ and $d=2.5 \mathrm{~cm}$ by $t$ test $(P<0.1)$. But Künnapas did not measure this underestimation.

Thus it was demonstrated that the amount of overestimation of the vertical line and the amount of underestimation of the horizontal line diminish correspondingly as the vertical line shifts from the midpoint position to the end position of the horizontal line. This fact must be taken into consideration in investigating the horizontal-vertical illusion. Then a further experiment is performed to examine this type of change of the apparent length of the horizontal line.

\section{Experiment 2}

Purpose. The purpose of this experiment is to clarify how the apparent length of the horizontal bisected line in the inverted $T$ figure changes with the variation of the length of the vertical line.

Condition. The length of the vertical line, which bisects the horizontal line as in Fig. 3, is varied in 9 steps : namely $1.25 \mathrm{~mm}, 2.5 \mathrm{~mm}$, $5 \mathrm{~mm}, 10 \mathrm{~mm}, 20 \mathrm{~mm}, 40 \mathrm{~mm}, 50 \mathrm{~mm}, 80 \mathrm{~mm}$, and $160 \mathrm{~mm}$.

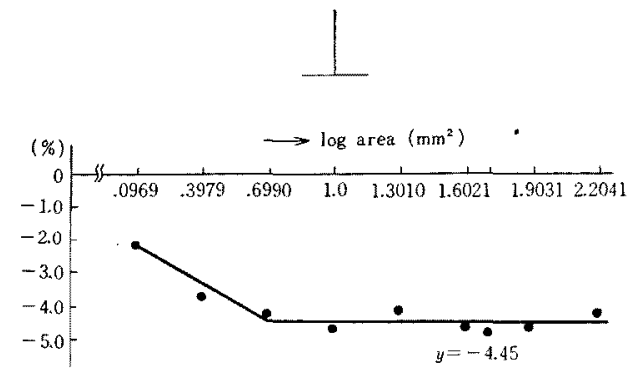

Fig. 3. The effect of the divided illusion of the horizontal line as a function of the area of the vertical line.

Results and discussion. The average amount of the divided illusion for $6 S \mathrm{~s}$ are shown in Fig. 3. The abscissa of Fig. 3 indicates the log area of the vertical line.

The amount of underestimation increases as the area or the length of the vertical line increases, for the width of the line is $1 \mathrm{~mm}$. But after its area reaches $5 \mathrm{~mm}^{2}$, the amount of underestimation becomes constant, regardless of the area of the vertical line. A horizontal line for the amount of the underestimation in the figure is fitted by the method of least squares $\left(r_{y x}=\right.$ .00288 is negligible).

\section{Experiment 3}

Purpose. The purpose of this experiment is to clarify how the apparent length of the bisected horizontal line in mutually bisected figure of vertical and horizontal lines varies with the change of the length of the vertical line.

Condition. The length of the vertical line shown in Fig. 4 is varied in 5 steps:

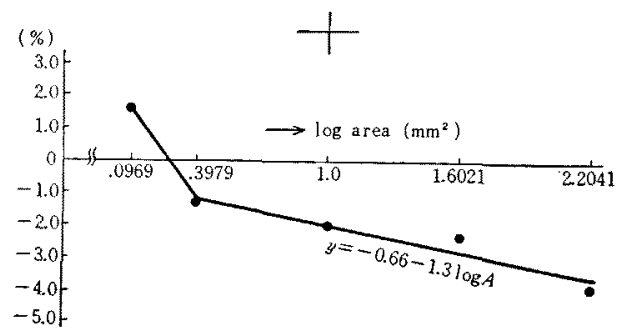

Fig. 4. The effect of the divided illusion of the horizontal line as a function of the area of the vertical line. 
namely, $1.25 \mathrm{~mm}, 2.5 \mathrm{~mm}, 10 \mathrm{~mm}, 40 \mathrm{~mm}$, and $160 \mathrm{~mm}$.

Results and discussion. Fig. 4 shows the average effects of the divided illusion of the horizontal line, obtained by $6 \quad S$ s. The abscissa of Fig. 4 indicates log area of the vertical line. Fig. 4 shows the following facts.

If the area of the vertical line is small, the horizontal line is overestimated, while if the area of the vertical line is large the horizontal line is underestimated and the amount of this underestimation increases linearly as $\log$ area of the vertical line increases. The line for the amount of the underestimation is fitted by the method of least squares $\left(r_{y x}=.996\right)$.

According to Obonai (1954, 1955) the distance between two dots, the distance which was bisected by a vertical line, changed its apparent distance in the following manner. If the dividing line was short the distance was overestimated, but as the length of the dividing line increased the amount of the overestimation decreased until finally it turned into the underestimation. The amount of underestimation gradually increased as the length of the line increased. Thus the present result corresponds to the Obonai's one, regardless of the difference between the types of the distance, filled and empty. Obonai $(1954,1955)$ explained this divided illusion by his theory of psychophysiological induction. Though his result was obtained in terms of the frequencies of the judgments, the present result can be explained by his theory. But a further analysis of details of the effect will be given with further research.

\section{Experiment 4}

Purpose. The purpose of this experiment is to clarify how the apparent length of the bisected vertical line changes with the variation of the length of the dividing horizontal line.

Condition. As Table 1 indicates a vertical line is bisected by a horizontal line or is un-

\section{Table 1}

The effect of the length of the bisecting horizontal line upon the apparent length of the vertical line.

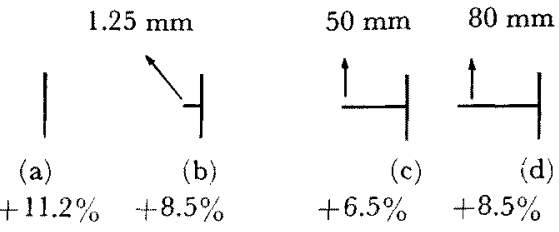

divided. The length of the horizontal line is varied.

Results and discussion. Table 1 shows the average amount of the overestimation of the vertical line, obtained by $6 S \mathrm{~s}$, as a function of the length of the dividing horizontal line. As Table 1 indicates the apparent length of the undivided vertical line is the longest of the four figures. And the apparent length of the vertical line does not vary so much with the variation of the length of the divided line. Thus the amount of overestimation of the vertical line, which is not combined with the horizontal line, was accurately measured.

Künnapas (1955) used the figure(c)shown in Table 1, and he obtained about $6 \%$ underestimation of the vertical as compared with the horizontal. But the author demonstrated the overestimation of the vertical line in the same figure by the different method from that of Kunnapas. Künnapas (1955) called the overestimation of the dividing line from his result. But by the author's method both the vertical and the horizontal lines are proved to be apparently lengthened in the figure (c) in Table 1. This experimental results will be mentioned later. In the figure $\vdash$, Künnapas (1955) and Ōno (1959) as well found the underestimation of the vertical line as compared with the horizontal. Thus the difference between the author's method and their methods, usually used up to the present, appears.

Thus it is possible to examine accurately the structure of the visual space by comparing the apparent length of the lines at different locations of visual field to the apparent length of a norm line. The 
author thinks that this is an important requisite for an approach to the horizontal-vertical illusion. So the next experiment is designed to see the structure of the visual space.

\section{Experiment 5}

Purpose. The purpose of this experiment is to clarify how the apparent length of a line varies with its directional location in the visual field.

Condition. A line of $50 \mathrm{~mm}$ is located at a position differing in directions, step by step by 30 degrees. The angle $\alpha$ of a line, shown in Fig. 5 , is varied in a random order of rotation of the line.

Results and discussion. The curves obtained by the average results for $6 S \mathrm{~s}$ are shown in Fig. 5. The abscissa of Fig. 5 shows the direction of the line. In the figure, $0^{\circ}$ position of the line means that it is located at a horizontal position. This line is used for a standard reference line in determining the apparent lengthening or shortening of the lines in many figures as mentioned in an early part of the article.

As Fig. 5 shows, the apparent length of a line changes regularly with its orientation. The curves in the figure are fitted by the method of least squares. The regression equation for a left parabola in the figure is

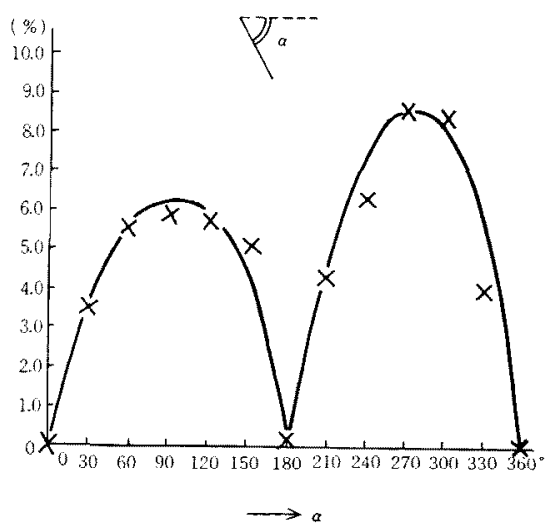

Fig. 5. The apparent length of a line as a function of its direction.

$$
y=-\frac{6.4}{8100} x(x-180),
$$

where $y$ is the relative amount of overestimation of a line, and $x$ is the angle $(x)$ of the line's direction in degree $\left(\sigma_{y x}=.192\right)$. And that for a right parabola is

$$
y=-\frac{8.7}{8100}(x-180)(x-360) \text {. }
$$

But this equation fits poor $\left(\sigma_{y x}=.194\right)$.

From Fig. 5 and the equations, it is found: a line looks longest when vertical and shortest when horizontal, while a tilted line looks longer than a horizontal line but shorter than a vertical line. Thus the Koffka's following description (1935) was clearly demonstrated. "The so-called overestimation of the vertical is a manifestation of the inequality of the horizontal and the vertical direction". Pollock and Chapanis (1952) performed a study similar to the present one. According to Pollock and Chapanis the lines tilted $20-30^{\circ}$ to the left of the vertical look longer than lines in any other orientation, and lines to the right of the vertical do not look as long as those tilted to the left of the vertical, though they covered experiments for only half the area of the visual field which was covered by the present study. Shipley and others (1949) previously showed that a line located in the region of 5 o'clock's position looks longest of the lines located at 3-6 o'clock, using the paired comparison. These results do not accord with the present result.

According to Delboeuf and others (Akishige, 1932) the upper half of the visual field is overestimated. But Akishige showed the following fact: the upper half of the visual field is not always overestimated, and if the stimulus object is located at a position higher than observer's eyes, the upper half of the object is overestimated, while if the object is located at a position lower than the observer's eyes, the lower half of the object is overestimated. Fischer also found the result reverse to the Delboeuf's one (Akishige, 1932). As Fig. 5 shows, the vertical line located at 
a position higher than the observer's eyes looks longer than the one located at a position lower than the observer's eyes. This result corresponds to the Delboeuf's. But it should be pointed out that the present experiment was performed under illuminated visual field. Akishige (1932) pointed out that the overestimation of the upper half is more remarkable in the lighted room than in the dark room. At any rate, the present experiment has verified the anisotropy of the visual space.

\section{Experiment 6}

Purpose. The purpose of this experiment is to investigate an interrelation between the apparent length of the line located at different direction and the divided illusion of the horizontal line.

Condition. The figure shown in Fig. 6 is presented. The direction of the titled line which bisects the horizontal line is varied. The angle $\alpha$ of the tilted lines shown in the figure is $187.5^{\circ}, 195^{\circ}, 210^{\circ}, 225^{\circ}, 240^{\circ}, 255^{\circ}$, or $270^{\circ}$. The apparent lengths of the tilted line and the horizontal line are measured respectively.

Results and discussion. Averages of $6 \mathrm{Ss}$ are plotted in Fig. 6. Four $S \mathrm{~s}$ out of 6 are the seam persons as in Experimant 5. The abscissa of Fig. 6 indicates the direction of the tilted line.

Fig. 6 shows that the dividing line is overestimated and its apparent length gradually increases as the angle $\alpha$ increases, and the vertical line looks longest. This increasing tendency is significant by H. B. Mann's test $(P<0.01)$. This result agrees with that shown in Fig. 5. On the other hand the apparent length of the divided horizontal line is shortened by bisection, but the amount of the apparent shortening decreases, on the whole, if the angle $\alpha$ decreases, and finally the line is overestimated at $187.5^{\circ}$ of $\alpha$. The upward tendency of the total curve plotted for the apparent lengths of the horizontal line is significant by Mann's test $(P<0.05)$. Then a further experiment is performed to examine the effect of direction of the

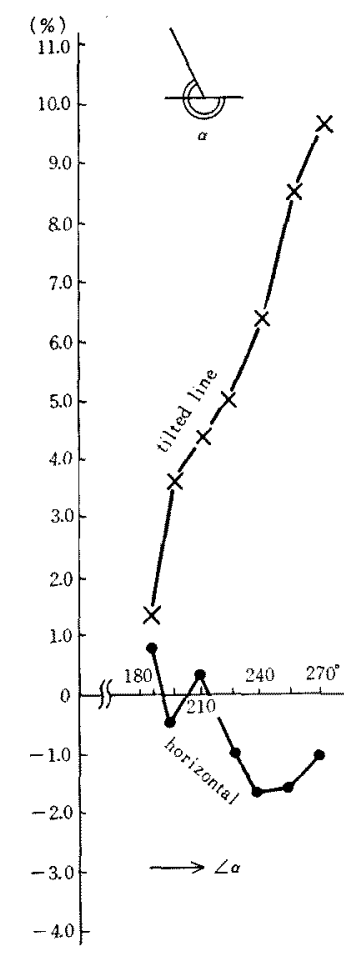

Fig. 6. The apparent lengths of the lines as functions of the direction of the tilted dividing line.

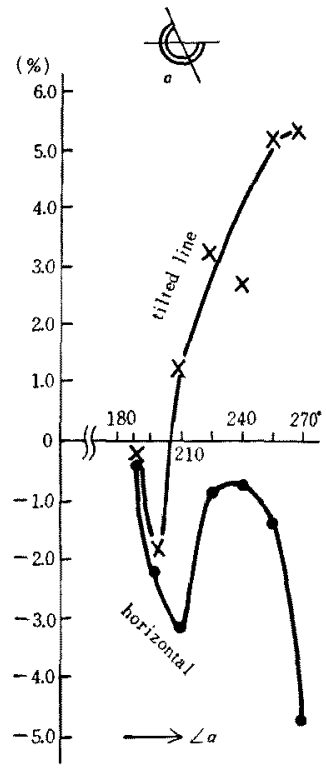

Fig. 7. The apparent lengths of the lines as functions of the direction of the tilted dividing line. line and the effect of bisection upon the apparent lengths of the lines.

\section{Experiment 7}

Purpose. The purpose of this experiment is to clarify the effects of the mutual bisection and the direction of a tilted dividing line upon the apparent lengths of the lines.

Condition. The figure shown in Fig. 7 is presented. The angle $\alpha^{\prime}$ 's of the tilted line are $187.5^{\circ}, 195^{\circ}, 210^{\circ}, 225^{\circ}, 240^{\circ}, 255^{\circ}$, and $270^{\circ}$.

Results and discussion. Average results for $4 S_{\mathrm{s}}$ are plotted in Fig. 7. As Fig. 7 shows, the amount of the overestimation of the vertical line is maximum. But this amount gradually decreases, on the whole, as the line inclines. This is the same tendency as that manifested in Fig. 5 and 6. But if the tilted line approaches the horizontal location, the line turns into 
the underestimation. The downward tendency of the total curve is significant by Mann's test $(P<0.01)$. Both two lines which are bisected mutually look shortened if the angle between them is small. The amount of the underestimation of the horizontal line is maximum if the dividing line is the vertical, diminishing when the dividing line inclines. This also agrees with the results shown in Fig. 6.

In Fig. 6 and 7 it may be remarkable that the mutual corresponding shiftings of the curves for overestimation and underestimation are found. This sort of interdependent modifications of the apparent lengths of the lines is further examined in the subsequent experiments.

\section{Experiment 8}

Purpose. The purpose of this experiment is to examine the effects of the mutual bisection upon the apparent lengths of lines and to examine the overestimation of the

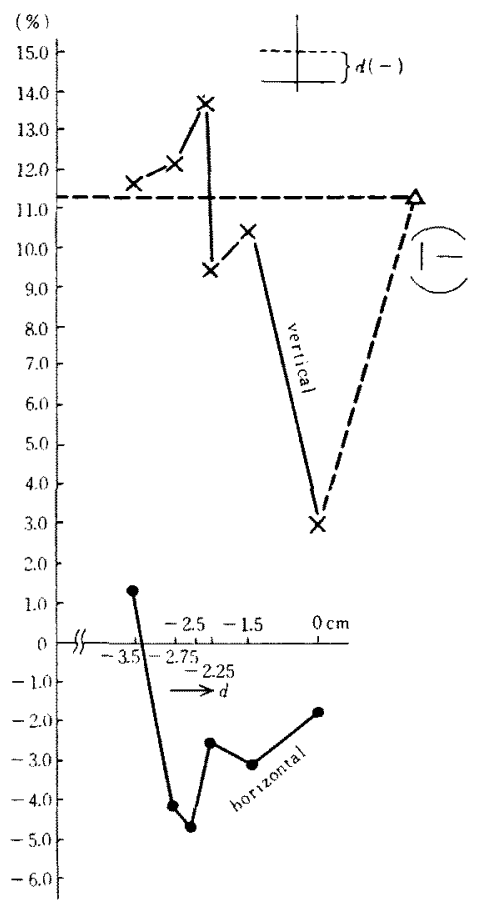

Fig. 8. The apparent lengths of the vertical and the horizontal line as functions of the position of the vertical line. vertical line.

Condition. The location of the vertical line, shown in Fig. 8, is varied in its height, keeping horizontal's height constant.

Results and discussion. Average results for $4 S \mathrm{~s}$ are indicated in Fig. 8. Fig. 8 shows nearly symmetrical two curves. The undivided vertical line is overestimated by about $11 \%$, but the amount of this overestimation is reduced to $3 \%$ if the vertical line is bisected by the horizontal. In other words the effect of the dividing or the shortening of the apparent length of the vertical line was demonstrated. The amount of the overestimation of the vertical line gradually increases as the vertical line shifts towards the higher position, and finally it reaches its maximum in the inverted $T$ figure. But it decreases again if the vertical separates from the horizontal. This decreasing was also shown by Ōno (1959), Takino (1956) and others.

In a corresponding manner to the above results, the amount of the underestimation of the horizontal is the minimum in the mutually bisected figure. It reaches its maximum in the inverted $\mathrm{T}$ figure, and further it turns into the overestimation if the vertical separates from the horizontal. The effect of the position of the dividing vertical line thus confirmed is also shown in Fig. 2.

Further, the curves in Fig. 8 show approximately symmetrical shapes. Ogasawara (1952) confirmed a fact similar to the above in the illusion of concentric circles. According to Ogasawara (1952) the curves for the relative amounts of overestimation of inner circles and those of underestimation of outer circles are symmetrical in their shapes, and the maximum overestimation of the inner circle is about $13 \%$. And maximum overestimation of the vertical line is about $14 \%$ in the present study (cf. Fig. 2, 8, 9, and 10). Further it is also about $14 \%$ in Künnapas' experiment (Künnapas, 1955). Ogasawara (1952) and the author have thus 


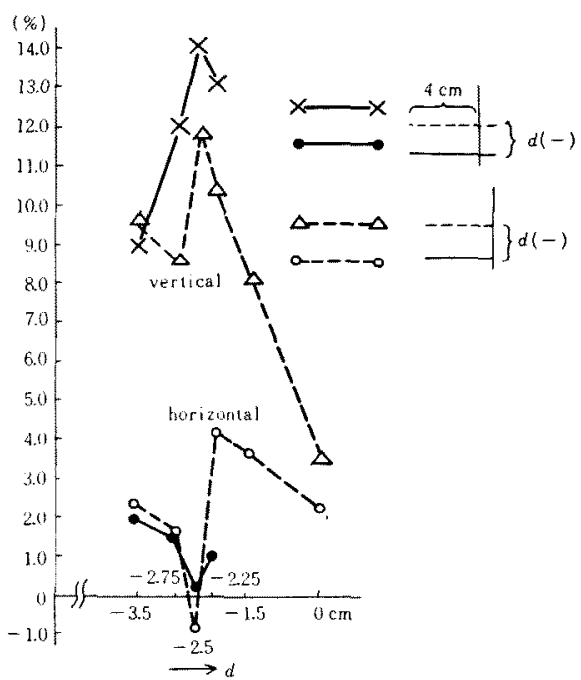

Fig. 9. The apparent lengths of the vertical and the horizontal line as functions of the position of the horizontal line.

confirmed an interdependent variation of the apparent extents of space in different optical illusions. Then a further experiment is performed to see the effect of the position of the vertical line.

\section{Experiment 9}

Purpose. The purpose of this experiment is to examine the effect of the position of the dividing vertical line upon the apparent lengths of the vertical and the horizontal lines.

Condition. The figures shown in Fig. 9 are presented and the position of the vertical line is varied as shown in Fig. 9.

Results and discussion. Fig. 9 shows the average results for $4 \mathrm{Ss}$. The four curves indicate the trend similar to that found in Fig. 8. But the overestimation of the horizontal line is remarkable in Fig. 9. This type of overestimation will again be shown in Experiment 13 (Fig. 13) where a consideration of this result will be given. But the following fact must be remarked: Künnapas (1955) obtained the underestimation of the vertical by the figures $\vdash$ and $\dashv$. Ono (1959) also obtained it by the former figure. But in the latter figure the apparent lengths of both the vertical and the horizontal lines actually lengthen, when measured by the author's method.

Künnapas (1955) pointed out that the following two different illusions appeared in the horizontal-vertical figures. a) The classical overestimation of the vertical as compared with a horizontal line of equal length, and b) the overestimation of the dividing line. The present experiment demonstrated b) type of illusion. This will be further shown in Experiment 12.

\section{Experiment 10}

Purpose. The purpose of this experiment is to examine how the apparent length of the divided vertical line changes with variation of the position of the dividing horizontal line.

Condition. The position of a horizontal line in the figure, shown in Fig. 10, is varied from the top to the bottom position of a vertical line.

Results and discussion. Fig. 10 indicates the average results for $6 \mathrm{Ss}$. The graph shows that the dividing effect or the apparent shortening of the vertical line is the maximum in the dividing at the middle portion of the vertical line. The difference

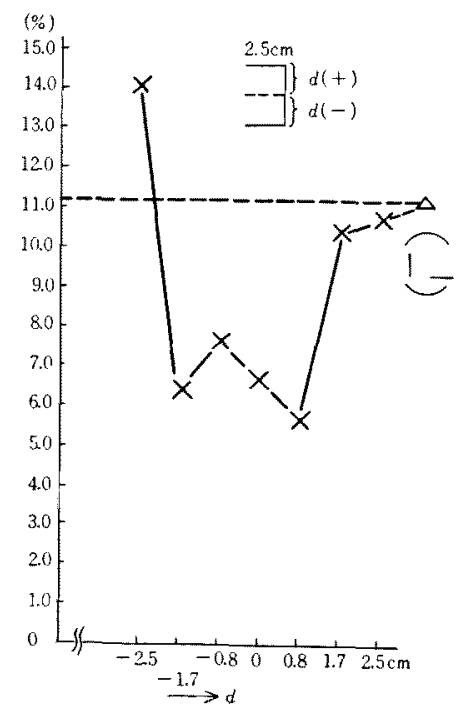

Fig. 10. The apparent length of the vertical line as a function of the position of the horizontal line. 
between the amounts of overestimation under the conditions of $d=-2.5$ $\mathrm{cm}$ and $d=-1.7 \mathrm{~cm}$ is significant by $t$ test $(P<0.025)$. Again the maximum overestimation of the vertical is about $14 \%$ as in Fig. 2, 8, and 9. This trend agrees with that shown in Fig. 8 and also with that obtained by Künnapas (1955).

The amount of the apparent shortening of the bisected vertical line $(4.5 \%)$, computed with reference to the horizontal dotted line in Fig. 10, approaches to that of the horizontal bisected line $(3.7 \%)$ shown in Fig. 3 . Comparing Fig. 8 and 10 , it might be proved that the shortening effect or the dividing effect by bisection is larger in the mutual bisected figure. But this can not be applied to Fig. 3 and 4 .

\section{Experiment 11}

Purpose. The purpose of this experiment is to investigate the effect of the position of a vertical line and the effect of the distance between the vertical and the horizontal lines upon their apparent lengths in the figure shown in Fig. 11.

Condition. The explanation is omitted, as the figures used can be understood by inspecting Fig. 11 and 12.

Results and discussion. The results are shown in Fig. 11 and 12. In Fig. 11 the results in the plus area of $d$ are the averages of $4 \mathrm{Ss}$, and the results in the minus area of $d$ are the averages of $5 \mathrm{Ss}$. Fig. 12 shows the average results for the same $5 S$ s as the above. Two $S$ s out of 5 or 4 participated under all conditions of Experiment 11.

In the right side of Fig. 11, the amount of overestimation of the vertical line decreases as the distance between the vertical and the midpoint of the horizontal increases. This trend was also indicated in Fig. 2. But $2 S$ s out of 4 are different persons from those in Experiment 1. The

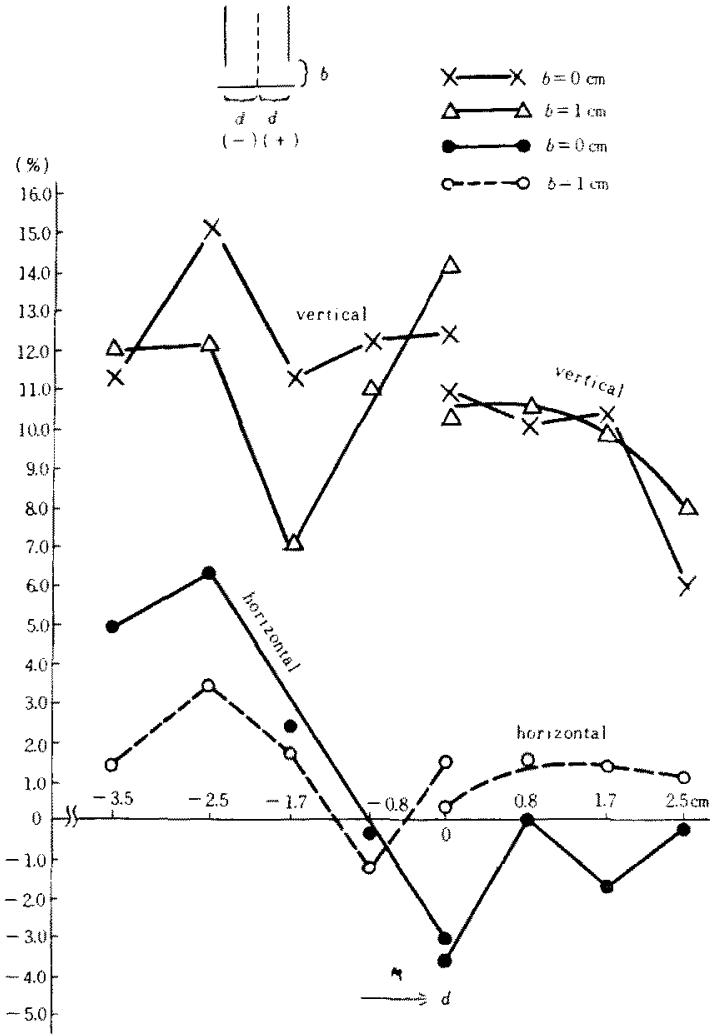

Fig. 11. The apparent lengths of the vertical and the horizontal line as functions of the position of the vertical line.

curves in the left side of the figure do not show this trend. If the vertical line is apart from the horizontal line, the latter is overestimated and this is also shown in Fig. 12, and the amount of the overestimation of the vertical line decreases as indicated in Fig. 12. Thus Fig. 12 shows that if the vertical separates from the horizontal, both the vertical and the horizontal curves approach each other. Besides, as is shown in Fig. 12, if the vertical is apart from the midpoint of the horizontal, both the vertical and the horizontal curves approach each other in their total shapes.

Thus the interdependent variations of the apparent lengths of the vertical and the horizontal were demonstrated by Fig. 12 as by Fig. 8 . In other words, the amount of apparent lengthening of the vertical 
and that of apparent shortening of the horizontal concomitantly vary. The figural process of the vertical line at the area which is apart from the horizontal by some distance is an antagonistic one to its figural process at the locus of the horizontal, and the horizontal may apparently lengthen. But it is uncertain how the processes behave.

In Fig. 4, 6, and 11, the overestimation of the divided horizontal line is indicated. This apparent lenthening of the divided line must also be remarked in investigating the horizontal-vertical illusion. The factor of the shortening or the lengthening of the apparent length of the divided line acts in the horizontal-vertical figures.

\section{Experiment 12}

Purpose. The purpose of this experiment is to analyze the overestimation of the dividing horizontal line in the $\rightarrow$ type of the figure.

Condition. The apparent length of the horizontal line is measured under varied conditions of the vertical line as shown in Fig. 13. The lines in four figures in Fig. 13 are bisected.

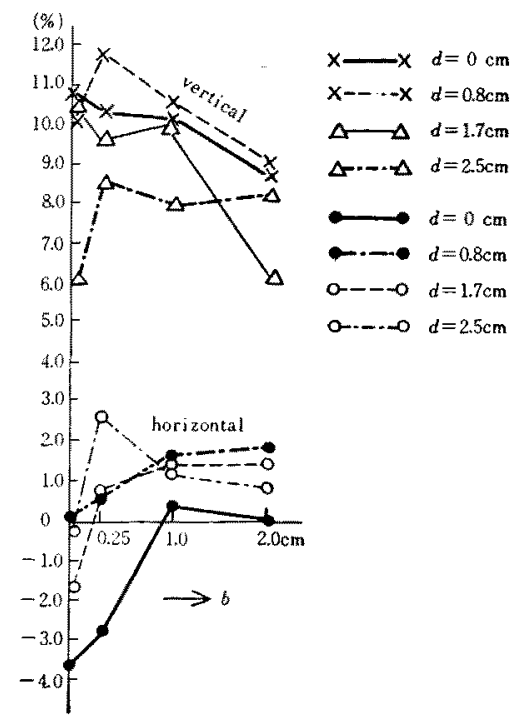

Fig. 12. The apparent lengths of the vertical and the horizontal line as functions of the position of the vertical line.
The observers are asked to observe the distance between the midpoints of the widths of the vertical lines.

Results and discussion. Fig. 13 shows the average results for $6 \mathrm{Ss}$. The abscissa of Fig. 13 indicates the added area of the vertical lines of each figure.

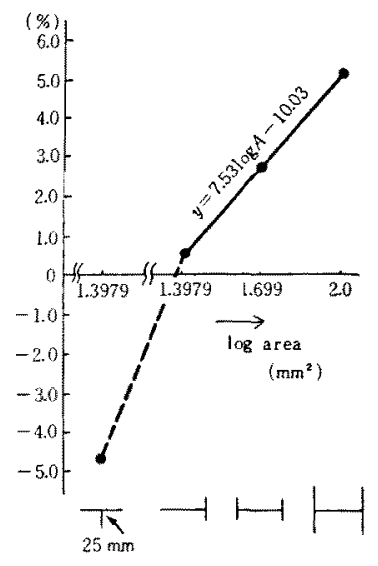

Fig. 13. The apparent length of the horizontal line as a function of the area of the vertical line.
The horizontal line in the figure $\mathrm{T}$ is underestimated by $4.7 \%$ and this amount may be said approximate to that in Fig. 3, though $4 S$ s out of 6 are different persons under both conditions. But if this vertical line is bisected by the horizontal line the horizontal is overestimated, though slight in amount. The significant difference was found for the results under these two conditions by $t$ test $(P<0.02)$.

The amount of overestimation of the horizontal line increases proportional to the logarithm of the added area of the vertical lines. The line shown in Fig. 13 is fitted by the method of least squares $\left(r_{y x}=.963\right)$. It was shown that the amount of overestimation of the horizontal dividing line depends upon the magnitude of the divided line. This overestimation of the horizontal was also shown in Fig. 9. And the overestimating tendency of the dividing vertical line under the figural condition $b=0 \mathrm{~cm}$ is shown in Fig. 11 and 12. The factor of overestimation of the dividing line pointed out by Künnapas (1955) was thus confirmed.

According to Kiesow (1906) the amount of underestimation of the horizontal increased as the verticals were lengthened in the figure $1-\mid$. Heymans proved the following fact in the Müller-Lyer illusion (Woodworth, 1954; Heymans, 1896): the 
amount of the illusion is proportional to the cosine of the angle between the oblique and the horizontal line, being zero when this angle is $90^{\circ}$. The above figure was called limiting figure of the Müller-Lyer figure by Lewis (1909). Lewis measured the apparent length of the horizontal of $50 \mathrm{~mm}$ in this figure as in the present study. $\mathrm{He}$ proved that the amount of underestimation of the horizontal decreases as the vertical line is varied in length from 5 to $35 \mathrm{~mm}$ on each side by an interval of $5 \mathrm{~mm}$, and ultimately, there occurs the overestimation of the horizontal line. This overestimation has also been confirmed by the present study. Obonai and Sugiuchi demonstrated by the above figure that the amount of underestimation of the horizontal increases as the verticals are lengthened (Shimada, 1952). But they used the figure in which the horizontal was $20 \mathrm{~mm}$ in length, and they varied the length of the verticals from 40 to $320 \mathrm{~mm}$. There are discrepancies between these results, and they are, probably, due to the differences between the procedures and the conditions of experiments differing in detailed points.

\section{Experiment 13}

Purpose. The purpose of this experiment is to analyze the variation of apparent length of the divided horizontal line in the different figural structures.

Condition. The apparent lengths of the horizontal lines shown in Table 2 are measured. The same instruction as in Experiment 12 is given for observing figures $d, e, f$, and $g$.

Results and discussion. Average results for $6 \mathrm{Ss}$ are shown under the figures in Table 2. Six $S$ s are the same persons as those in Experiment 12 (Fig. 13). Each vertical line in the figures in Table 2 is $25 \mathrm{~mm}$ in its length. The results obtained by seven figures can be classified into the following three groups in terms of the figural structures: 1) the figures a and b, 2) the figures $c$ and $d, 3$ ) the figures $e, f$, and $\mathrm{g}$.

\section{Table 2}

The effect of the figural structure upon the apparent length of the horizontal line.

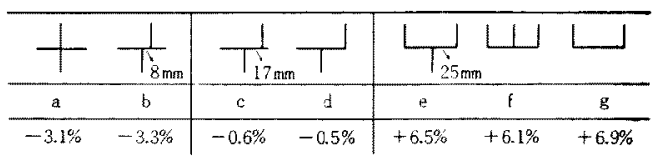

The considerations of the figures a, d, and $g$ and of the results obtained by them are given here chiefly, for these figures are typical in their figural structures in each group of the figures. In the figure a, or the mutual bisected figure, the horizontal line is underestimated. The amount of this underestimation diminishes in the figure $d$, and the apparent change of the horizontal is nearly null. But a considerable amount of overestimation of the horizontal line appears in figure g. But the areas of the extra vertical lines are held constant in the three figures a, d, and $g$.

It was proved that the apparent length of the horizontal line depends upon the area of the vertical in Experiment 12, while it was proved that the apparent length of the horizontal depends upon the structure or the configuration of the figure in $\mathrm{Ex}$ periment 13. In the figure $\mathrm{d}$, however, the effect of the two vertical lines seems to act antagonistically. The bisecting vertical line acts to shorten the apparent length of the horizontal line as was confirmed in the present study. On the other hand the apparent lengthening of the horizontal occurs in the figure $J$ as was demonstrated above. So both the effects of apparent shortening and lengthening of the horizontal may counteract each other, thus the horizontal in the figure d hardly changes its apparent length. Ikeda (1956) found the counteracting effects of the two concentric inspection circles, investigating the figural after-effects, upon the apparent extent of the test circle which is intermediate in its extent between two inspection circles. In this case both enlargening and shrinkagening effects upon 
the test figure by two figures also counteract as in the present case. The figure $g$ is the added figure of the figures, and $L$. So the considerable amount of overestimation of the horizontal may appear additively. Besides, the vertical appears longer than the horizontal of equal length in this sort of figural configuration. The square does not appear equal in its vertical and its horizontal line (Fröbes, 1917; Rausch, 1952). According to Rausch (1952) the vertical is overestimated by about $5 \%$ as compared with the horizontal. The apparent lengths of the horizontals in the figures $a, b, c, d$, and $g$ changed as if they were pulled by the vertical lines.

But the structure of the figures $e$ and $f$ are complicated. It looks as if the figural processes of the vertical lines at the end positions of the horizontal act to inhibit or to eliminate the effect of the bisected vertical line. There appears approximately an equal amount of overestimation of the horizontal to that in the figure $g$.

\section{Summary}

The present study was designed to investigate the interdependence of the horizontal-vertical illusion and the illusion of divided line. The analytical study was performed to clarify the interdependence of the overestimation of the vertical line and the divided illusion by many figures. The apparent lengths of the vertical and the horizontal line were measured. The main results can be summarized as follows:

1. The amount of overestimation of the vertical line decreases linearly as the distance between the vertical line and the midpoint position of the horizontal line increases in the inverted $T$ figure, with some exceptions.

2. The horizontal line is underestimated in the inverted $T$ figure, and the amount of the underestimation decreases if the vertical line is apart from the midpoint of the horizontal. In the inverted $T$ figure, the amount of this underestimation increases as the area (or the length) of the vertical line increases, and after reaching a certain value it becomes constant.

3. In the mutual bisected figure of a vertical line and a horizontal line, the latter is overestimated if the area of the vertical line is small. But as this area increases, it turns into the underestimation whose amount increases proportional to the logarithm of the area of the vertical line.

4. The horizontal line bisected by a tilted line is also underestimated.

5. The apparent length of a line varies regularly with its direction. It is maximum when the line is located at the vertical position, but it decreases as the line inclines, reaching minimum at a horizontal position. This variation of the apparent length follows the parabola. Moreover, Delbouef's illusion was demonstrated.

6. The apparent length of a vertical divided by a horizontal line is minimum by bisection. As the horizontal dividing line shifs from the midpoint position to the end position of the vertical, the apparent length of the vertical increases.

7. The apparent length of the horizontal modifies with a corresponding trend to the variation of the apparent length of the vertical.

8. In the figure $\dashv$, both the horizontal dividing and the vertical divided line are overestimated and the amount of overestimation of the horizontal increases proportional to the logarithm of the added area of the vertical divided lines.

9. Where the vertical line is separated from the horizontal line, the horizontal is overestimated.

10. The factor of overestimation of the dividing line in the horizontal-vertical figures, pointed out by Künnapas, was confirmed.

11. The factor of the shortening and occasionally the lengthening of the apparent length of the divided line act in the horizontal-vertical figures.

12. The illusions studied were con- 
sidered with reference to other visual phenomena.

\section{REFERENCFS}

Akrshige, Y. Experimentalle Untersuchungen über die sogenannte Überschätzung des oberen Sehfeldes. Jap. 7. Psychol., 1932, 7, 203-225.

Fröвes, J. S. Lehrbuch der experimentellen Psychologie. Bd. 1, 1917.

Heymans, G. Quantitative Untersuchungen über das " optische Paradoxon". Z. Psychol., 1896, 9, $221-255$.

IKEDA, H. On the cancellation of two opposing figural after-effects. Jap. 7. Psychol., 1956, 26, 407-410.

ImaI, S. A review of recent studies on geometrical optical illusion. fap. 7. Psychol, $1960,30,366-375$.

KIEsow, F. Über einige geometrisch-optische Täuschungen. Arch. ges. Psychol., 1906, 6, 289-305.

KOFFкA, K. Principles of gestalt psychology. 1935, p. 275.

Künnapas, T. M. An analysis of the "verticalhorizontal illusion". 7. exp. Psychol., 1955, 49, 134-140.

Lewis, E. O. Confluxion and contrast effects in the Müller-Lyer illusion. Brit. 7. Psychol,
$1909,3,21-41$.

ObonaI, $T$. Induction effects in estimates of extent. 7. exp. Psychol., 1954, 47, 57-60.

ObonaI, T. Shichikaku (Visual perception). Tokyo: Nakayama Shoten, 1955.

Ogasawara, J. Displacement-effect of concentric circles. Jap. 7. Psychol., 1952, 22, 224-234.

ONo, S. A study on the horizontal-vertical illusion. Jinbunkenkyu, 1959, 10, No. 5, 41-60.

Pollock, W. T., \& Chapanis, A. The apparent length of a line as a function of its inclination. Quart. F. exp. Psychol., 1952, 4, 170-178.

RAUsch, E. Struktur und Metrik figural-optischer Wahrnehmung. 1952, p. 175.

Shimada, K. Survey of the studies on MüllerLyer illusion. Jap. J. Psychol., 1952, 23, 111123.

Shipley, W. G., Nann, Barbara M., \& Penfield, MARY J. The apparent length of tilted lines. 7. exp. Psychol, 1949, 39, 548-551.

Takino, C. An experimental study on the horizontal-vertical illusion. Abstract of report on 20 th Congress of the F. P. A., 1958.

Woodworth, R. S., \& Schlosberg, H. Experimental psychology. 1954.

(Received July 7, 1960) 